\title{
Transperineal single-port robot-assisted radical prostatectomy with Si da Vinci surgical system: initial experience and description of technique
}

\author{
Kangxin Ni", Dingwei Xue", Gonghui Li \\ Department of Urology, Sir Run Run Shaw Hospital, Zhejiang University School of Medicine, Hangzhou, China \\ Contributions: (I) Conception and design: All authors; (II) Administrative support: G Li; (III) Provision of study materials or patients: D Xue; (IV) \\ Collection and assembly of data: K Ni; (V) Data analysis and interpretation: D Xue; (VI) Manuscript writing: All authors; (VII) Final approval of \\ manuscript: All authors. \\ "These authors contributed equally to this work. \\ Correspondence to: Gonghui Li. Department of Urology, Sir Run Run Shaw Hospital, Zhejiang University School of Medicine, No. 3 East Qingchun \\ Road, Hangzhou 310016, China. Email: 3193119@zju.edu.cn.
}

\begin{abstract}
Background: Single-port robotic-assisted radical laparoscopic prostatectomy has emerged as a novel robotic-assisted radical laparoscopic prostatectomy in recent years, arousing wide attention. However, singleport robotic-assisted radical laparoscopic prostatectomy using Si da Vinci surgical system has been rarely reported, especially via the transperineal approach.

Methods: We retrospectively collected 9 cases of prostate cancer patients who underwent transperineal single-port robot-assisted radical prostatectomy ( $t$-spPARP) using Si da Vinci surgical system in our center from May 2020 to June 2020. The operation time, estimated blood loss (EBL), complications, changes in prostate-specific antigen (PSA) 3 months after surgery, and urinary continence recovery 6 months after surgery were analyzed.

Results: No perioperative complications were recorded. The median [interquartile range (IQR)] operation time was 350 [150] min and the median [IQR] EBL was 300 [100] mL. PSA levels were less than $0.01 \mathrm{ng} / \mathrm{mL}$ at 3 months postoperatively in all cases (undetectable in 8 cases). All the 9 patients recovered their urinary continence 6 months after surgery and merely two patients required pads during the day.

Conclusions: t-spRARP was verified as a safe and feasible surgical alternative to treat patients with localized prostate cancer, especially for those whose prostate is small-volume or who had abdominal surgery history.
\end{abstract}

Keywords: Transperineal; single-port; radical prostatectomy; prostate cancer; minimally invasive surgery

Submitted May 24, 2021. Accepted for publication Sep 22, 2021.

doi: $10.21037 /$ tcr-21-898

View this article at: https://dx.doi.org/10.21037/tcr-21-898

\section{Introduction}

The proposal and popularization of minimally invasive concepts and the continuous improvement of laparoscopic instruments have enabled radical prostatectomy (RP) to enter a new field from traditional radical retropubic prostatectomy (RRP) to laparoscopic radical prostatectomy (LRP). Schuessler et al. first reported LRP in 1997 (1), marking that the surgical treatment of prostate cancer has entered the era of minimally invasive surgery. However, laparoscopic surgery is more difficult to perform. With narrow operating space, lack of tactile feedback, and difficulty in pelvic floor sutures, LRP greatly extend the operation time and did not show an obvious advantage in tumor control, urinary control, and sexual function at the early stage. In the following years, the continuous improvement and development of surgical techniques and 
instruments made LRP accepted by more and more patients as well as surgeons, which began to promote LRP on a large scale.

In 2001, Abbou et al. reported the robot-assisted laparoscopic radical prostatectomy (RARP) for the first time (2), which opened the prelude to RARP in the surgical treatment of prostate cancer. The advent of the da Vinci surgical system once again put prostate cancer surgery in a new stage of minimally invasive surgery. Compared with traditional laparoscopy, the da Vinci surgical system has a 3D field of view with steady and flexible robot arms, which greatly improves the surgeon's operational ability and enables surgeons to accurately identify anatomical structures and levels during the surgery. Besides, the da Vinci system also accelerates the speed of learning and growth for the reduced difficulty of the operation to a certain extent. Nowadays, RARP has become the main surgical method of prostate cancer treatment in Europe and North America. From 2003 to 2007, the proportion of minimally invasive procedures (including LRP and RARP) for RP in the United States rose sharply from $4.9 \%$ to $44.5 \%$, while RRP dropped from $89.4 \%$ to $52.9 \%$ (3). So far, many studies have suggested that RARP can achieve positive surgical margins (PSM) rate as low as LRP, and it has shown advantages over LRP in intraoperative blood loss $(188.0 \mathrm{~mL})$, intraoperative blood transfusion rate $(1.8 \%)$, and average postoperative hospital stay (4 days) $(4,5)$. While improving the incidence of perioperative complications, RARP reduces the proportion of long-term urinary incontinence to about $9 \%$, which is better than open surgery [odds ratio $(\mathrm{OR})=1.53$ ] and $\mathrm{LRP}(\mathrm{OR}=2.39)(6)$.

The conventional RARP establishes channels through 4 Trocars so that the four robotic arms of the surgical robot can enter the surgical operation space, thus leaving four wounds postoperatively. With the increasing demand of patients for no scars or fewer scars after surgery, single port laparoscopic surgery has been widely favored by more and more urologists and patients due to its advantages in achieving good surgical results while reducing the number of surgical incisions and scars. Therefore, singleport robotic-assisted radical laparoscopic prostatectomy (spRARP) came into being. Recently, a new platform da Vinci single-port (SP) system (Intuitive Surgical Inc., Sunnyvale, CA, USA) was introduced, whose articulating instruments was operated via a single access. The SP system is promising for robotic surgery while is unavailable in China and many other countries nowadays. On the other hand, it has been recently reported that transperitoneal
spRARP was well applied in prostate cancer patients with the use of a single port and the conventional da Vinci Si robotic instruments (7). Herein, we report the preliminary experience of 9 transperineal spRARP ( $\mathrm{t}$-spRARP) cases that were performed in our hospital and intend to provide a new reference for spRARP performed in the da Vinci Si robotic platform.

We present the following article in accordance with the STROBE reporting checklist (available at https://dx.doi. org/10.21037/tcr-21-898).

\section{Methods}

\section{Data source and patient selection}

We retrospectively collected 9 cases of prostate cancer patients who were performed t-spRARP in our hospital from May 2020 to June 2020. During this period, two surgeons in our team collected preoperative, intraoperative and postoperative data, and another colleague collected prognostic data. The study was conducted in accordance with the Declaration of Helsinki (as revised in 2013) and was approved by Institutional Review Board (Sir Run Run Shaw Hospital, Medicine School, Zhejiang University, 20190218-34) and individual consent for this retrospective analysis was waived. All transrectal prostate biopsy diagnoses of the included patients were prostate adenocarcinoma. All the t-spRARP cases were performed by an experienced surgeon (Gonghui $\mathrm{Li}$ ) with 600 cases of experience of multiport RARP.

\section{Inclusion criteria and variables definition}

Exclusion criteria for patients: prostate weight $>80 \mathrm{~mL}$, body mass index $(\mathrm{BMI})>35 \mathrm{~kg} / \mathrm{m}^{2}$, multiple biopsies and critical patients. Preoperative evaluation factors are including age at surgery, BMI, biopsy Gleason score, and prostate-specific antigen (PSA) level.

\section{Endpoints and data collected}

In this study, we aimed to report the preliminary experience of $t$-spRARP and describe the perioperative results and follow-up related to this novel robotic-assisted surgical procedure. We, therefore, collected intraoperative data including total operation time and estimated blood loss (EBL), postoperative pain scores (range from 0 to 10) at 8,12 , and $16 \mathrm{~h}$ after t-spRARP, complications, and 


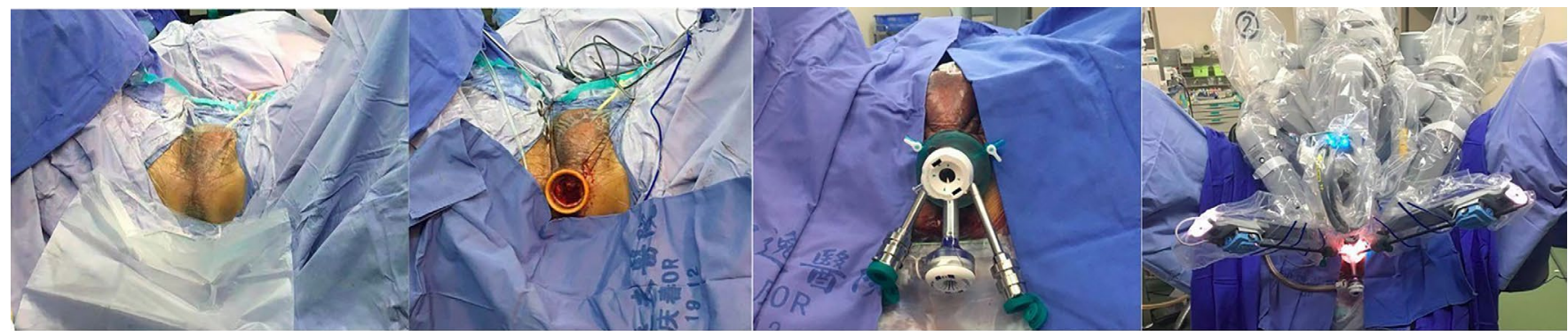

Figure 1 Trocar placement for transperineal single-port robot-assisted radical prostatectomy using da Vinci Si platform.

readmissions. The postoperative follow-up mainly included continence and PSA level at 3 months postoperatively.

\section{Statistical analysis}

All the data were collected and analyzed using the Statistical Package for the Social Sciences (SPSS). Continuous variables were described as medians and interquartile ranges (IQRs) and categorical variables as frequencies and proportions.

\section{da Vinci Si robot implementation and technical modifications}

The da Vinci surgical system was utilized to perform the surgery, and the 9 operations were performed using the "Si" model docking using a three-arms configuration. The following robotic instruments were equipped: $12 \mathrm{~mm} 30^{\circ}$ up scope, Hot Shears monopolar curved scissors, and ProGrasp forceps (Intuitive Surgical Inc., Sunnyvale, CA, USA).

\section{Trocar placement and docking technique}

The patients were placed in an exaggerated lithotomy position with a $20^{\circ}$ Trendelenburg tilt. The perineal incision was made at the apex of a semicircular line $(6 \mathrm{~cm})$ extending between the ischial tuberosities, the subcutaneous fascia tissue was separated, and the central tendon was then incised along with the rectal and urethral muscles were cut. The instruments enter into the anterior space of the rectum by blunt separation, followed by levator ani muscle pushed aside and Denonvilliers' fascia exposed. A single port was subsequently inserted with a surgical wound protector (Nantong angel medical instruments Co., Ltd., China) surrounding it. After the pneumoperitoneum establishment, the lens along with two robotic arms and an auxiliary operating hole were installed or fixed through the wound protector (Figure 1).

\section{da Vinci transperineal spRARP procedure}

After the apex of the prostate and the urethra were exposed, both sides of the prostate were bluntly separated extending to the bladder neck. Denonvilliers' fascia was transversely cut apart beneath the prostate, and was continuously separated forwards and downwards close to the prostate, and seminal vesicles and vas deferens Ampulla were then exposed and dissociated with the ampulla of the vas deferens cut off. The lateral prostatic ligaments were ligated and incised on both sides. Subsequently, the urethra was isolated and incised at the junction of the apex of the prostate and the urethra. The prostate was then separated to the bladder neck pulled by the apex of the prostate. Finally, the bladder neck was incised and the prostate was removed. 2-0 absorbable thread was used to suture the urethra and bladder neck continuously (Figure 2).

\section{Results}

\section{Patient demographics}

The median [IQR] age of the patients was 65 [3] years, the median [IQR] BMI was $24.5[4.4] \mathrm{kg} / \mathrm{m}^{2}$. The preoperative PSA level of 3 patients was $<10 \mathrm{ng} / \mathrm{mL}$ while 4 patients' preoperative PSA level ranged from 10 to $20 \mathrm{ng} / \mathrm{mL}$ and 2 patients' preoperative PSA level beyond $20 \mathrm{ng} / \mathrm{mL}$. The biopsy pathology reported 33\% Grade Group (GrGP)1, 33\% GrGP2, 22\% GrGP3, 11\% GrGP4, 0\% GrGP5. The median [IQR] prostate size was 29.9 [10.1] mL (Table 1).

\section{Intraoperative}

The median total operative time [IQR] reported was 350 

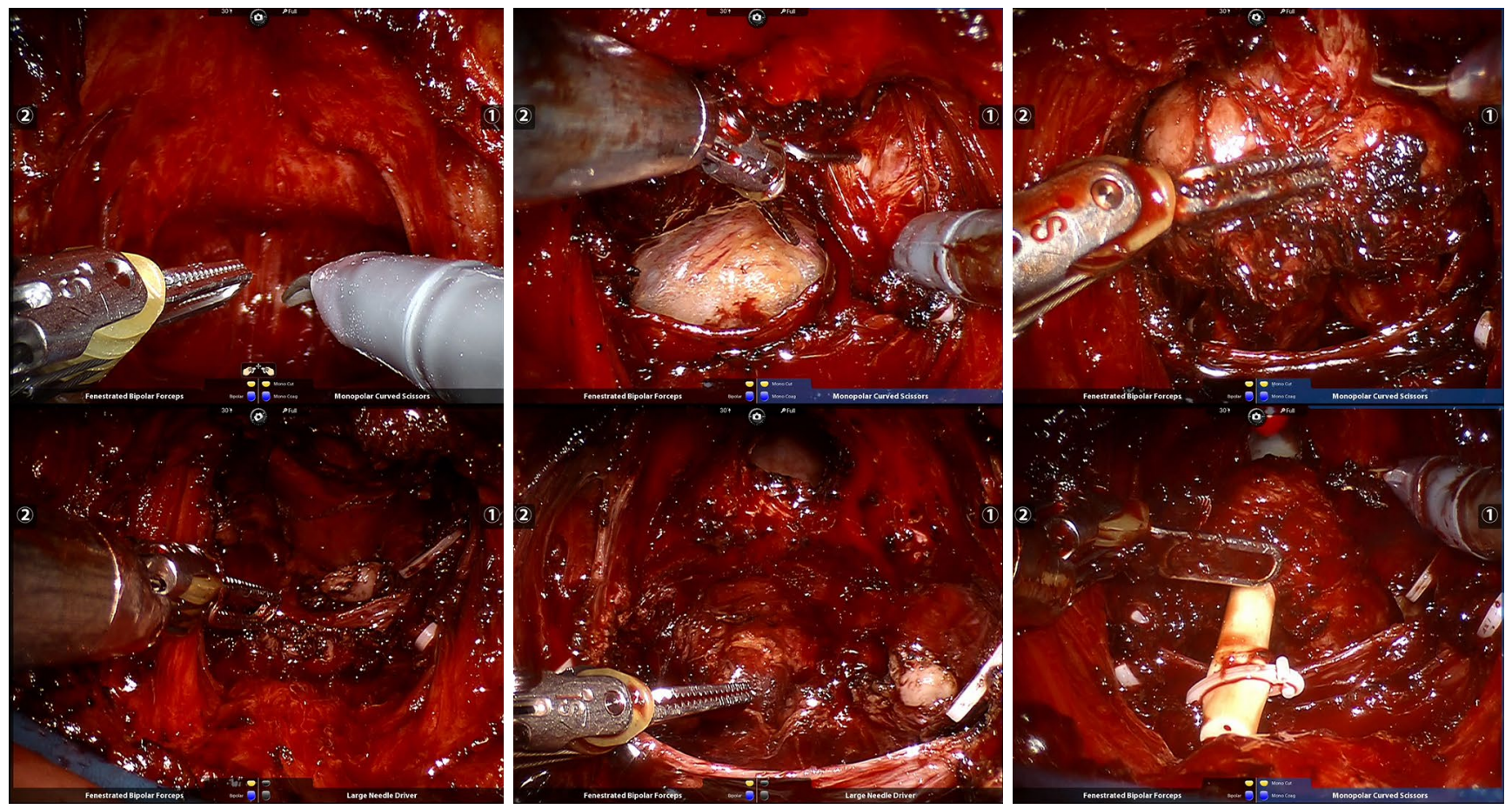

Figure 2 Intraoperative view during the transperineal single-port robot-assisted radical prostatectomy.

Table 1 Patients' demographics

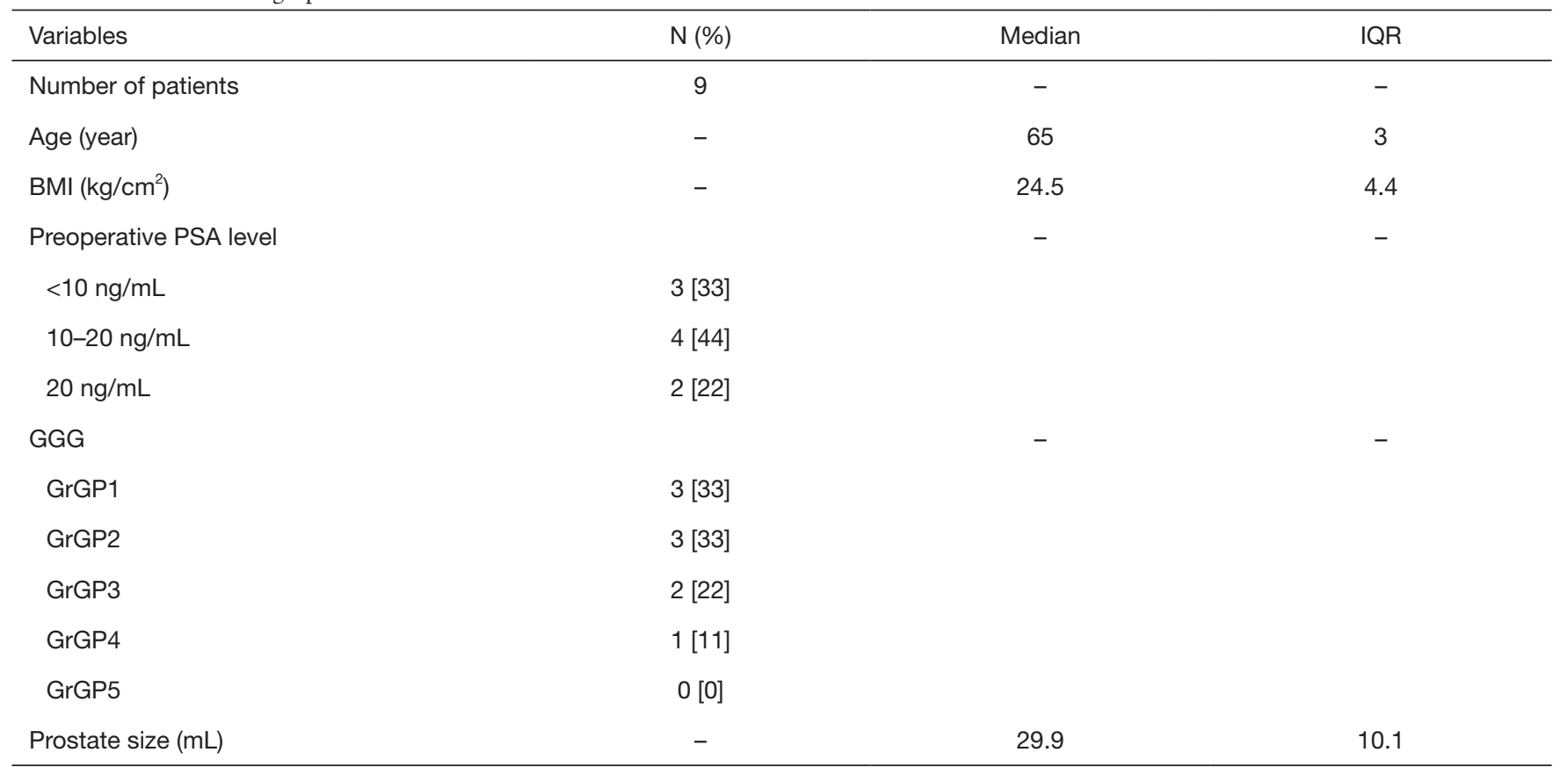

IQR, interquartile range; BMI, body weight index; PSA, prostate specific antigen; GGG, Gleason Grade Group. 
[150] min, and median EBL [IQR] was 300 [100] mL (Table 2). Importantly, both the total operative time and EBL were remarkably decreased as the surgical proficiency improved (Figure 3). Besides, there are neither intraoperative complications nor blood transfusions during the surgery.

\section{Postoperative and pathological outcomes}

The median [IQR] hospital length of stay of these 9 patients was 7 [2] days (Table 3). The median pain scale score reported at 8,12 , and $16 \mathrm{~h}$ after spRARP was 1.9 , 1.4 and 1, respectively. Four patients experienced a slow incision healing process (the wound was unhealed during the hospital stay) and recovered after regular dressing in outpatient. The final pathology reported 33\% GrGP1, 33\% GrGP2, 22\% GrGP3, 0\% GrGP4, 11\% GrGP5. In all, only one patient $(11 \%)$ had a GrGP upgrading when comparing the final pathology with the previous biopsy report. Importantly, none of the 9 patients exhibited PSM.

\section{Follow-up (Table 4)}

The PSA level of the 8 patients decreased to $0 \mathrm{ng} / \mathrm{mL}$ and one patient's PSA level was merely $0.01 \mathrm{ng} / \mathrm{mL}$. Continence outcomes were collected at our outpatient department

Table 2 Intraoperative data

\begin{tabular}{lcc}
\hline Variables & Median & IQR \\
\hline Total operative time $(\mathrm{min})$ & 350 & 150 \\
EBL $(\mathrm{mL})$ & 300 & 100 \\
\hline
\end{tabular}

Values are presented as median and IQR. EBL, estimated blood loss; IQR, interquartile range.

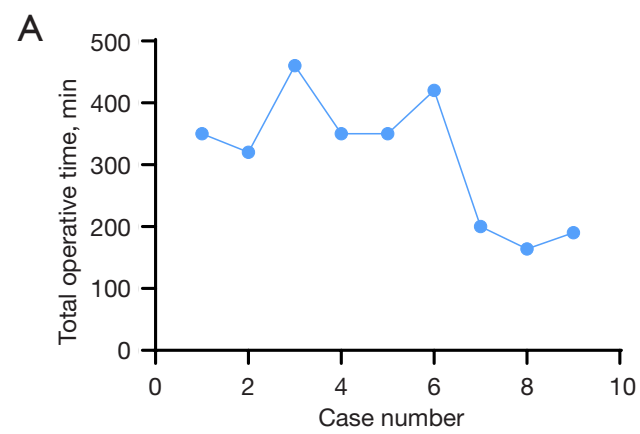

6 months after t-spRARP. In all, 7 patients (78\%) recovered full continence and $2(22 \%)$ patients used pads during the day.

\section{Discussion}

Single-port robotic-assisted surgery was generally defined as robot-assisted surgery with only one skin incision. In the early days, the spRARP was referred to as the RARP with multiple independent small holes under a single incision. However, this type of operation was accompanied by more fascia injuries and longer incision healing time (8). Kaouk et al. first introduced an

Table 3 Postoperative data

\begin{tabular}{lccc}
\hline Variables & N [\%] & Median & IQR \\
\hline Length of stay (days) & - & 7 & 2 \\
Pain scale score at 8h postoperatively & - & 2 & 0 \\
Pain scale score at12 h postoperatively & - & 2 & 1 \\
Pain scale score at 16 h postoperatively & - & 1 & 0 \\
Poor incision healing & $4[44]$ & - & - \\
GGG & & - & - \\
GrGP1 & $3[33]$ & & \\
GrGP2 & $3[33]$ & & \\
GrGP3 & $2[22]$ & & \\
GrGP4 & $0[0]$ & & \\
GrGP5 & $1[11]$ & & - \\
Gleason upgrading from biopsy & $1[11]$ & - \\
PSM & $0[0]$ & - \\
\hline
\end{tabular}

IQR, interquartile range; PSM, positive surgical margins; GGG, Gleason Grade Group.

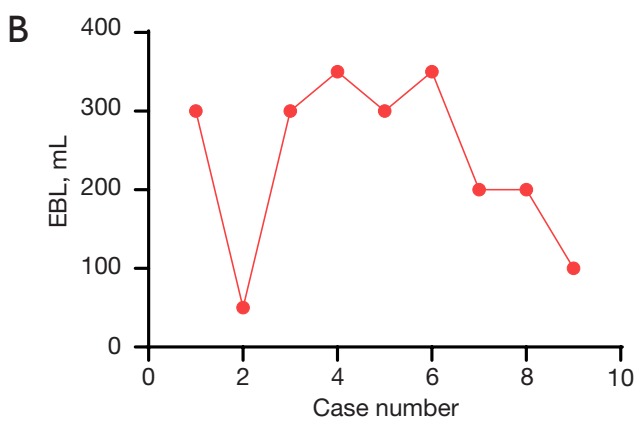

Figure 3 Total operative time (A) and EBL (B) for transperineal single-port robot-assisted radical prostatectomy. EBL, estimated blood loss. 
Table 4 Follow-up data

\begin{tabular}{ll}
\hline Variables & $\mathrm{N}[\%]$ \\
\hline Postoperative PSA level (3 months) & \\
$\leq 0.01 \mathrm{ng} / \mathrm{mL}$ & $9[100]$ \\
Continence after 6 months & \\
Full continence (no pads) & $7[78]$ \\
With pads in the day & $2[22]$ \\
\hline
\end{tabular}

initial experience of two RARP cases using a transperitoneal approach through a trans-umbilical incision with the new single-port platform, that is, da Vinci SP system (9). More recently, Kaouk et al. used the da Vinci SP surgical system in 10 consecutive RARP cases via the extraperitoneal approach and achieved satisfactory outcomes including operative time, complications, hospital stay length, and continence recovery (10). Though the single-port platform shows promising potential for surgical breakthroughs, it has not yet been available in any medical center in China. As for most medical centers without da Vinci SP platform, it is a big challenge to achieve a true single-port approach RARP with the straightened robotic arm on account of limited operating space, and operational flexibility. Chang et al. reported for the first time that transperitoneal spRARP was successfully performed by optimizing surgical techniques based on the traditional da Vinci surgical platform using the Si HD model (7).

As another important route for performing RP (11-14), the perineal approach could achieve a more direct anatomic approach through a relatively small and intimate incision. However, the narrow operative narrow, the position of the prostate in the pelvis and ergonomic issues challenging the operating surgeon performed RP via the perineal approach and have obstacled its application while the introduction of the robotic system alleviating difficulties as mentioned above in radical perineal prostatectomy (RPP). Therefore, it was also promising to investigate the feasibility of the spRARP through the transperineal approach based on the traditional da Vinci Si surgical platform.

Significantly, t-spRARP using da Vinci Si surgical platform was successfully performed in this report and achieved similar outcomes compared with the traditional multi-port RARP. All the patients in this study were carefully selected and analyzed. The median [IQR] EBL was 350 [150] mL, which was close to our conventional multi-port RARP. The median [IQR] total operative time was 300 [100] min, which was longer compared with the durations reported in the previous meta-analysis (15) due to the initial practice of surgeons and the surgical history of patients (one-third of patients had abdominal surgical history). Although our present study does not include a comparison among different approaches, we can use this study as a reference for the transperineal RARP. With the surgical experience accumulating, the operative time and EBL were expected to decrease.

Kaouk et al. reported 4 cases performing RARP via single-port robotic perineal approach, and surgical margins were focally positive in 3 patients with complex surgical histories (16). In our series, given our initial experience with t-spRARP, PSMs were not reported in all 9 cases including the 3 cases with abdominal history, owing to our careful dissection. As for incision healing, 4 patients in our study suffered poor healing for their large volume prostate and prolonged operation time, which indicated that $\mathrm{t}$-spRARP will be not an appropriate method for a prostate cancer patient whose prostate is large. Focusing on urinary function, 8 patients have regained full urinary continence 6 months after surgery, while only one patient needs pads during the day. As was defined by Takenaka et al., anterior support from the puboprostatic collar will avoid incontinence in an immediate time postoperatively induced by bladder mobilization (17).

We also recognize some limitations to our initial practice: it might be essential to extend the incision for the extraction if the prostate is too large. Besides, the cohort of patients in this study was small, the intraoperative data was incomplete, and the advantages of this technique were not validated by comparing it with other approaches. All the disadvantages could be mainly attributed to the learning curve for the use of a novel surgical method.

In all, our preliminary application experience exhibited that the technique of t-spRARP is feasible and oncologically safe. However, it should be noted in this study that we carefully selected patients with lower BMI, and carried out careful preoperative preparation, including digital rectal examination, prostate imaging evaluation, incision, and port location design, etc. The console surgeon and assistant also have extensive experience in multi-port RARP. Through the initial practice, we draw a preliminary conclusion that the early learning curve does not greatly affect the safety and efficacy of patients. However, we recommend a thoughtful and cautious attitude and method for the adoption and learning curve of this new surgery procedure due to the lack of wrist surgical instruments and instrument traction. 
Besides, the setting of the exclusion criteria for this report made it impossible for this study to evaluate the feasibility of this procedure for more challenging operations. As this study is still in the preliminary application stage, it is currently unable to achieve long-term follow-up of the prognosis of the procedure. Therefore, we will consider including a prospective study of long-term functional recovery and prostate cancer outcome related to this procedure in further studies.

\section{Conclusions}

The $9 \mathrm{t}$-spRARP cases in this report have finally achieved satisfactory outcomes. Meanwhile, it can satisfy patients' higher demands for the aesthetics of the postoperative scar to the greatest extent due to its privacy intimate incision. Moreover, t-spRARP seems to a good alternative for those patients who had small volume prostate or abdominal history. However, this technology still demands more application experience to improve the surgical technique as well as standardize and simplify the surgical procedure. Besides, we need further analyze the learning curve, perioperative complications, long-term functional recovery, and comparison with conventional RARP as the cases accumulating to some extent.

\section{Acknowledgments}

We acknowledge the assistance of Prof. Jochen Neuhaus to polish our manuscript.

Funding: This study was supported by National Natural Science Foundation of China (81672520, 81870484), Science and Technology Planning Project of Zhejiang (2019C03089), Zhejiang Medical and Health Science and Technology Plan Project (WKJ-ZJ-2031).

\section{Footnote}

Reporting Checklist: The authors have completed the STROBE reporting checklist. Available at https://dx.doi. org/10.21037/tcr-21-898

Data Sharing Statement: Available at https://dx.doi. org/10.21037/tcr-21-898

Conflicts of Interest: All authors have completed the ICMJE uniform disclosure form (available at https://dx.doi. org/10.21037/tcr-21-898). The authors have no conflicts of interest to declare.

Ethical Statement: The authors are accountable for all aspects of the work in ensuring that questions related to the accuracy or integrity of any part of the work are appropriately investigated and resolved. The study was conducted in accordance with the Declaration of Helsinki (as revised in 2013). The present study was approved by the Ethics Committee of Sir Run Run Shaw Hospital, School of Medicine, Zhejiang University (IRB number: 2019021834). Written informed consent was obtained from all the patients.

Open Access Statement: This is an Open Access article distributed in accordance with the Creative Commons Attribution-NonCommercial-NoDerivs 4.0 International License (CC BY-NC-ND 4.0), which permits the noncommercial replication and distribution of the article with the strict proviso that no changes or edits are made and the original work is properly cited (including links to both the formal publication through the relevant DOI and the license). See: https://creativecommons.org/licenses/by-nc-nd/4.0/.

\section{References}

1. Schuessler WW, Schulam PG, Clayman RV, et al. Laparoscopic radical prostatectomy: initial short-term experience. Urology 1997;50:854-7.

2. Abbou CC, Hoznek A, Salomon L, et al. Laparoscopic Radical Prostatectomy with a Remote Controlled Robot. J Urol 2017;197:S210-2.

3. Kowalczyk KJ, Levy JM, Caplan CF, et al. Temporal national trends of minimally invasive and retropubic radical prostatectomy outcomes from 2003 to 2007: results from the 100\% Medicare sample. Eur Urol 2012;61:803-9.

4. Lowrance WT, Eastham JA, Savage C, et al. Contemporary open and robotic radical prostatectomy practice patterns among urologists in the United States. J Urol 2012;187:2087-92.

5. Wang Y, Gieschen H, Greenberger M, et al. Survival After Robotic-Assisted Prostatectomy for Localized Prostate Cancer: An Epidemiologic Study. Ann Surg 2019. [Epub ahead of print].

6. Novara G, Ficarra V, Mocellin S, et al. Systematic review and meta-analysis of studies reporting oncologic outcome after robot-assisted radical prostatectomy. Eur Urol 2012;62:382-404.

7. Chang Y, Lu X, Zhu Q, et al. Single-port transperitoneal 
robotic-assisted laparoscopic radical prostatectomy (spRALP): Initial experience. Asian J Urol 2019;6:294-7.

8. Leewansangtong S, Vorrakitkatorn P, Amornvesukit T, et al. Laparo-endoscopic single site (LESS) robotic radical prostatectomy in an Asian man with prostate cancer: an initial case report. J Med Assoc Thai 2010;93:383-7.

9. Kaouk J, Bertolo R, Eltemamy M, et al. Single-Port Robot-Assisted Radical Prostatectomy: First Clinical Experience Using The SP Surgical System. Urology 2019;124:309.

10. Kaouk J, Valero R, Sawczyn G, et al. Extraperitoneal single-port robot-assisted radical prostatectomy: initial experience and description of technique. BJU Int 2020;125:182-9.

11. Ficarra V, Novara G, Artibani W, et al. Retropubic, laparoscopic, and robot-assisted radical prostatectomy: a systematic review and cumulative analysis of comparative studies. Eur Urol 2009;5 5:1037-63.

12. Wirth MP, Hakenberg OW. Surgery and marketing: comparing different methods of radical prostatectomy. Eur Urol 2009;55:1031-3.

Cite this article as: Ni K, Xue D, Li G. Transperineal single-port robot-assisted radical prostatectomy with $\mathrm{Si}$ da Vinci surgical system: initial experience and description of technique. Transl Cancer Res 2021;10(11):4694-4701. doi: $10.21037 /$ tcr-21-898
13. Wroński S. Radical perineal prostatectomy - the contemporary resurgence of a genuinely minimally invasive procedure: Procedure outline. Comparison of the advantages, disadvantages, and outcomes of different surgical techniques of treating organ-confined prostate cancer (PCa). A literature review with special focus on perineal prostatectomy. Cent European J Urol 2012;65:188-94.

14. Nargund VH, Zaman F. Radical prostatectomy--too soon to abandon the perineal approach? Nat Rev Urol 2011;8:179-80.

15. Kallidonis P, Rai BP, Qazi H, et al. Critical appraisal of literature comparing minimally invasive extraperitoneal and transperitoneal radical prostatectomy: A systematic review and meta-analysis. Arab J Urol 2017;15:267-79.

16. Kaouk JH, Akca O, Zargar H, et al. Descriptive Technique and Initial Results for Robotic Radical Perineal Prostatectomy. Urology 2016;94:129-38.

17. Takenaka A, Tewari AK. Anatomical basis for carrying out a state-of-the-art radical prostatectomy. Int J Urol 2012;19:7-19. 